\title{
Sudden Unexpected Deaths due to Multiple Metastasis of Colon Cancer: With a Focus on Cardiac and Dural Metastasis
}

Joo-Young $\mathrm{Na}^{1}$, Hee Joo Kwon ${ }^{1}$, Jin-Haeng $\mathrm{Heo}^{2}$, Young-Il Park ${ }^{2}$, Sang-Beom Im $^{2}$

${ }^{1}$ Department of Pathology, Pusan National University Yangsan Hospital, Yangsan, Korea, ${ }^{2}$ Forensic Medicine Division, National Forensic Service Busan Institute, Yangsan, Korea

Received: July 6, 2021

Revised: July 26, 2021

Accepted: August 2, 2021

\section{Correspondence to} Joo-Young $\mathrm{Na}$ Department of Pathology, Pusan National University Yangsan Hospital, 20 Geumo-ro, Mulgeumeup, Yangsan 50612, Korea Tel: +82-55-360-1864

Fax: +82-55-360-1865

E-mail:pdrdream@gmail.com
A malignancy is a fatal condition that could occur through various mechanisms. Forensic pathologists sometimes find unexpected findings during autopsy and post-mortem (PM) tests. Colorectal cancer is one of the leading causes of cancer-related deaths worldwide. The deceased was a 64-year-old man with a medical history of right hemicolectomy due to colon cancer approximately two years earlier. He was found dead at his home. He was admitted to the hospital due to subdural hemorrhage (SDH) two days prior to his demise and was discharged without the permission of the doctor after one day of hospitalization. An autopsy was performed within two days of his death. After gross dissection, the cause and manner of death were assumed to be SDH and unnatural death, respectively. Microscopic examination revealed fresh SDH and dural metastasis of signet-ring cell carcinoma. Furthermore, metastasis was identified in the heart, stomach, and peritoneum. Immunohistochemical examination revealed cancer cells to originate from the colon. After meticulous PM examination, including gross dissection, microscopic examination, PM computed tomography, and PM laboratory tests, the cause and manner of death were determined as SDH and natural death, respectively. This case report highlights the importance of comprehensive PM evaluation for investigating death.

Key Words: Subdural hematoma; Dura mater; Neoplasm metastasis; Colorectal neoplasms; X-ray computed tomography

\section{Introduction}

An autopsy is an important method of investigating cases of death. Forensic pathologists determine the cause and manner of death through meticulous autopsy, including various laboratory tests using post-mortem (PM) samples. Sometimes, both autopsy and PM tests had unexpected findings. Colorectal cancer is one of the three most commonly diagnosed malignancies worldwide and is a leading cause of cancer-related death [1]. Malignancy could cause death in individuals through various mechanisms, including cachexia, direct local mass effect, metastasis to vital organs, and so on. Herein, we present a case of sudden unexpected death due to subdural hemorrhage (SDH) in a man with multiple metastasis, including the dura and the heart, incidentally revealed through microscopic examination after gross dissection. This case could be helpful to forensic pathologists who should be considered from the perspective of forensic pathology.

\section{Case Report}

The deceased was a 64-year-old man who was found dead at his home. He had a medical history of right hemicolectomy and was on chemotherapy for colon 
cancer approximately two years ago. He complained of a headache and was admitted to the emergency room two days prior to his death. After a medical evaluation, he was diagnosed with SDH and admitted to the intensive care unit. However, he was discharged upon his own request without permission from the doctor after one day of hospitalization.

An autopsy was performed within two days after death with a court-issued warrant at the request of the public prosecutor. External examination revealed no injuries other than the needle marks that were generated during hospital admission. On internal examination, a focal subgaleal hemorrhage was noted on the left parietal area, and SDH was identified on both sides of the cerebral hemisphere (Fig. 1). A thickened gastric wall was identified with dimensions of approximately $1.0 \times 0.5 \mathrm{~cm}$, and approximately 1-mm-sized tiny nodules were also identified on the peritoneum nearby anastomosis site due to the previous right hemicolectomy. The examination of the other internal organs was unremarkable. After gross dissection, the cause and manner of death were assumed to be SDH and unnatural death, respectively.

PM computed tomography (PMCT) showed SDH on both sides of the cerebral hemisphere, and the amount of hemorrhage was greater on the left side. A midline deviation to the right side was noted, and the left side of the lateral ventricle was not observed
(Fig. 2). Microscopic examination of the dura showed fresh SDH and neoplastic cells on the epidural side. These neoplastic cells contained abundant intracytoplastic mucin, which pushed the nucleus to the periphery, adopting the appearance of a signet ring. Furthermore, these signet-ring cancer cells were observed in the myocardium of the heart, stomach, and peritoneum. The mucosal lesion was vague, and both serosal involvement and vascular invasion by cancer

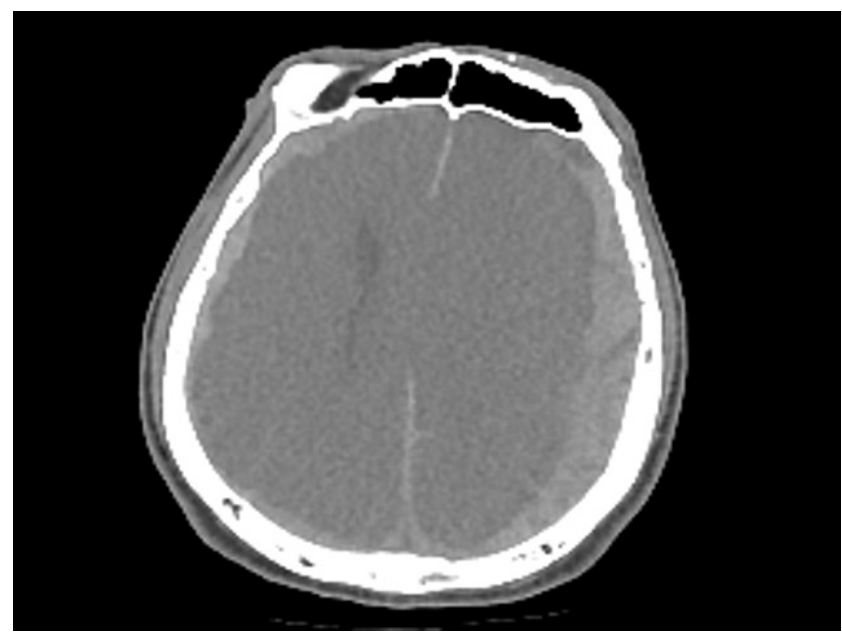

Fig. 2. Post-mortem computed tomography shows subdural hemorrhage on both sides of the cerebral hemisphere and the amount of hemorrhage is greater on the left side. A midline deviation to the right side was observed and the left side of the lateral ventricle was not observed.

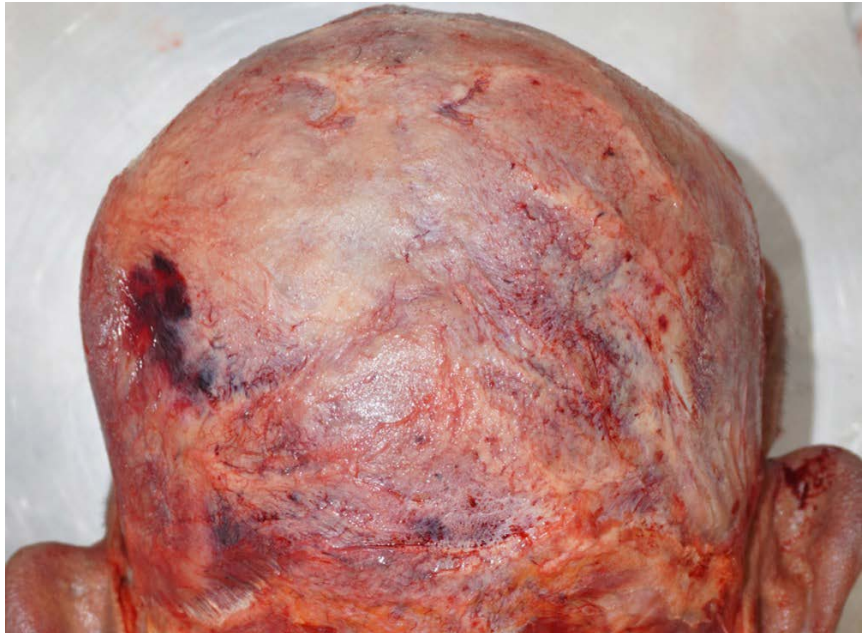

A

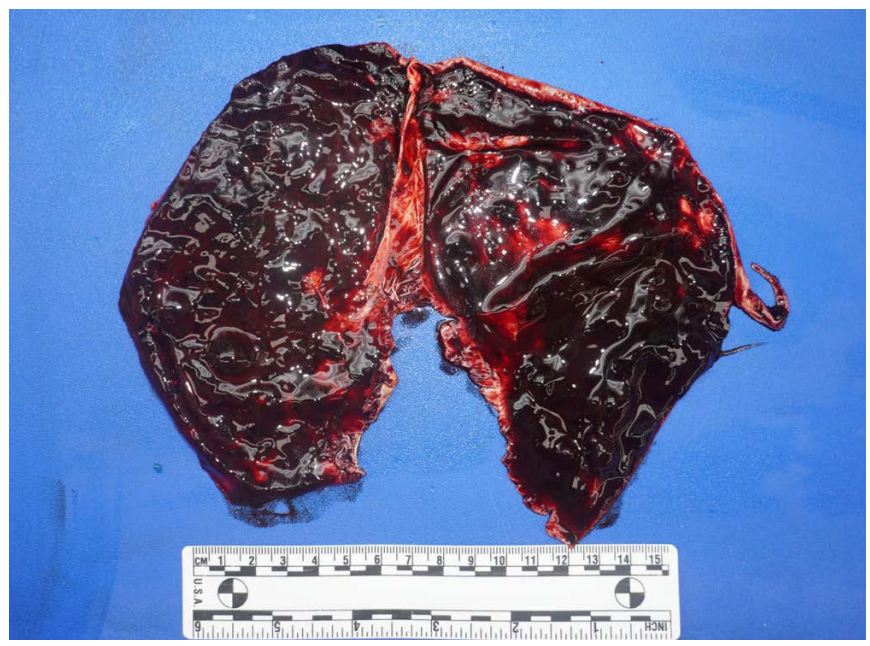

B

Fig. 1. A focal subgaleal hemorrhage is noted on the left parietal area (A). Subdural hemorrhage is noted on both sides of the cerebral hemisphere (B). 
cells were noted in the stomach. Immunohistochemical examination revealed that these cancer cells were positive for both cytokeratin (CK) 20 and CDX-2, and they were negative for CK7 (Fig. 3). Toxicological tests using PM blood showed no significant findings (several drugs were identified with blood concentrations within the therapeutic ranges, and the blood alcohol concentration was less than $0.010 \%$ ). Biochemical analysis of the vitreous humor revealed no remarkable findings. The glycated hemoglobin level was $5.0 \%$ (reference range, less than 6.5\%), and the blood ketone level was unremarkable.

After comprehensive PM examination, including gross dissection, microscopic examination, PMCT, and PM laboratory tests, the authors concluded that the deceased died of SDH in a setting of multiple metastasis of signet-ring cell colon cancer. Furthermore, the authors decided that SDH was caused by dural metastasis of signet-ring cell colon cancer. Finally, the manner of death was determined to be natural.

\section{Discussion}

The common sites of metastasis from colorectal cancer are the lymph nodes, liver, and lungs. Autopsy studies have shown that the incidence of cardiac metastasis from colorectal cancer is $1.4 \%$ to $7.2 \%$ [2]. To date, however, only 12 cases of cardiac metastasis from colon cancer have been reported in the English literature [3]. Tumors could reach the heart via three pathways: hematogenous spread, lymphatic spread, and direct extension. Spread by the hematogenous route generally gives rise to myocardial or endocardial metastasis and is common in melanoma, lymphoma, and sarcoma. Spread by the lymphatic route often results in pericardial and epicardial tumor involvement, as with many epicardial tumors such as cancers of the lung and breast. Direct extensions could be divided into two pathways; the first is the transvenous extension. This is a pathway through the inferior vena cava; for example, certain tumors such as renal cell carcinoma and hepatocellular carcinoma could extend into the inferior vena cava and grow into the right atrium via transvenous extension. The other is a direct extension of the surrounding organs and tissues. Locally aggressive mediastinal and pleural tumors, such as mesothelioma, can directly invade the pericardial sac [4]. In this case, metastatic cancer cells were located in the interstitium of the myocardium, and vascular invasion by cancer cells was noted in the stomach. Therefore, we thought that this metastasis originated from a hematogenous route. The pericardium is the most frequently involved site of cardiac metastasis, comprising $64 \%-69 \%$ of all cardiac metastasis [5,6]. Epicardial and myocardial involvement represent the second and third most common sites of cardiac metastasis, 25\%-34\% and 29\%-32\%, respectively $[5,6]$. Endocardial and intracavitary metastasis are rare, accounting for $3 \%-5 \%$ of cardiac metastasis on autopsy [5,6]. Of these metastatic locations, epicardial and myocardial metastasis can show some clinical manifestations, such as atrial and ventricular arrhythmia, congestive heart failure with systolic and diastolic dysfunction, myocardial ischemia from perivascular coronary artery compression, tumor embolism or coronary invasion, and cardiac rupture. Unfortunately, the pericardium was not histologically examined in this case; however, the pericardium was grossly unremarkable. In this case, cardiac metastasis occurred in the myocardium; however, there was no cardiac-specific clinical manifestation, and this cardiac metastasis was incidentally identified during PM microscopic examination. The deceased showed no cardiac symptoms, and cardiac metastasis was not decided as a cause of death in the present case. However, forensic pathologists should be aware that cardiac metastasis could be present in various cardiac symptoms, including sudden cardiac death.

Unfortunately, the colon was not opened during gross dissection; however, no abnormalities, such as a palpable mass or a thickened wall, were identified during gross dissection. Although the authors could not evaluate a primary colon cancer, they decided that multiple metastasis originated from the colon cancer through histologic and immunohistochemical examinations. The patient underwent right hemicolectomy and received chemotherapy two years ago; however, late metastasis from colorectal carcinoma was previously reported [7].

The mechanism of dural metastasis is most often through hematogenous spread or direct invasion from cranial metastasis, although a direct extension to the 

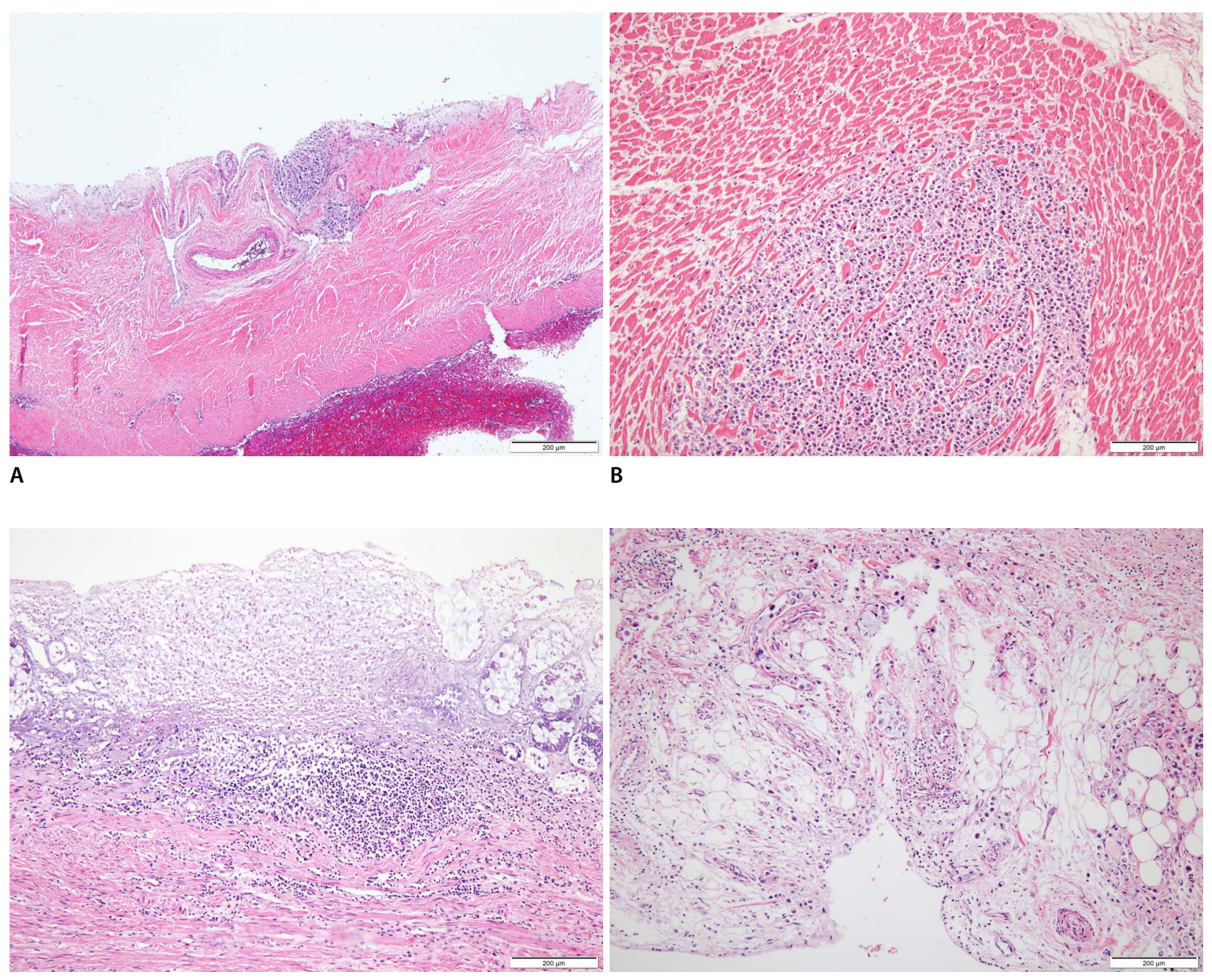

C

D

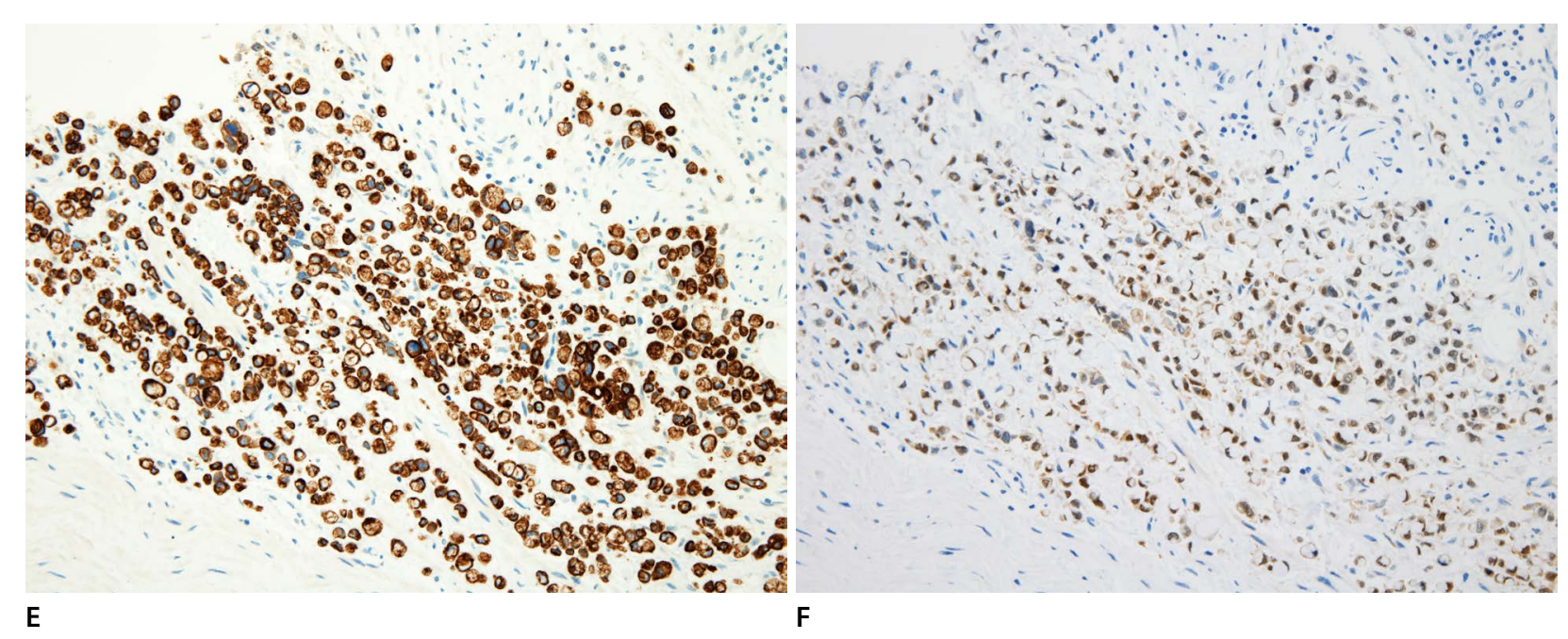

Fig. 3. Microscopic examination of the dura reveals fresh subdural hemorrhage and metastasis of the signet-ring cell carcinoma on the epidural side $(A, H \& E, \times 40)$. Signet-ring cancer cells are also noted in the myocardium $(B, H \& E, \times 100)$. Post-mortem change is identified in the mucosa, and signet-ring cancer cells are noted in the submucosa of the stomach. However, the mucosal infiltration by cancer cells is uncertain (C, H\&E, $\times 100)$. Cancer cells infiltrate through the serosa of the stomach, and vascular invasion is also noted (D, H\&E, $\times 100)$. Immunohistochemical examination reveals that cancer cells originated from colon cancer ( $E$, cytokeratin 20, ×200; F, CDX-2, ×200). 
dura from a cerebral parenchymal metastasis might rarely occur [8]. Head trauma is a well-known cause of SDH; however, SDH associated with dural metastasis has also been reported [9]. Furthermore, a case of a dural metastatic adenocarcinoma in an individual with recent head trauma and SDH was reported [10]. The mechanisms of development of SDH due to dural metastasis include the rupture of blood vessels formed by neo-vascularization of the hemorrhage, obstruction of dural venous vessels leading to dilation and rupture, or a pre-existing SDH serving as a medium for the deposition of blood-borne metastasis [8]. The most common sources of metastasis to the dura are the prostate, breast, lung, and stomach cancers [8].

After gross dissection, the cause and manner of death were assumed to be SDH and unnatural death, respectively. Microscopic examination of the dura revealed metastatic signet-ring cell carcinoma. Furthermore, immunohistochemical examination revealed that these signet-ring cancer cells originated from colon cancer. These findings indicate the possibility of the development of SDH due to dural metastasis, which means that death, in this case, is a result of the disease. Furthermore, it seemed that the severity and extent of the parietal subgaleal hemorrhage were not sufficient to cause fatal SDH. Although the relative amount of SDH between both sides could not be evaluated during gross dissection, PMCT indicated that the amount of hemorrhage was greater on the left side and indicated right-side deviation of the midline and loss of the left lateral ventricle. This leftside predominant hemorrhage did not correspond to a contrecoup injury because subgaleal hemorrhage was noted on the left side. Of course, a final falling down could occur during an agonal stage, and the subgaleal hemorrhage on the left parietal area with less amount of the right side SDH could develop due to a final falling, as shown on the PMCT.

Microscopic examination revealed the cause of death in $2 \%$ of cases where histology had been undertaken, and it added to the cause of death in $8 \%$ of cases, confirming the macroscopic findings in $61 \%$ of cases in the previous report [11]. Aut-opsy literally means self and seeing; in other words, it means an eye witness [12]. Gross dissection is undoubtedly the basis of an autopsy, and autopsy begins with gross dissection. Furthermore, with a comprehensive approach that includes microscopic examination, PM imaging, and PM laboratory tests, the cause and manner of death could be further clarified beyond visible autopsy findings.

ORCID: Joo-Young Na: https://orcid.org/0000-0003-1138433X; Hee Joo Kwon: https://orcid.org/0000-0001-79681694; Jin-Haeng Heo: https://orcid.org/0000-0002-39408615; Young-Il Park: https://orcid.org/0000-0003-21875618; Sang-Beom Im: https://orcid.org/0000-0002-92784679

Conflicts of Interest Joo-Young $\mathrm{Na}$, a contributing editor of the Korean Journal of Legal Medicine, was not involved in the editorial evaluation or decision to publish this article. All remaining authors declare that there is no conflict of interest.

\section{Acknowledgments}

This study was supported by a 2021 research grant from Pusan National University Yangsan Hospital.

\section{References}

1. Siegel RL, Miller KD, Fuchs HE, et al. Cancer statistics, 2021. CA Cancer J Clin 2021;71:7-33.

2. Mukai K, Shinkai T, Tominaga K, et al. The incidence of secondary tumors of the heart and pericardium: a 10-year study. Jpn J Clin Oncol 1988;18:195-201.

3. Tsujii Y, Hayashi Y, Maekawa A, et al. Cardiac metastasis from colon cancer effectively treated with 5 -fluorouracil, leucovorin, and oxaliplatin (modified FOLFOX6) plus panitumumab: a case report. BMC Cancer 2017;17:152.

4. Goldberg AD, Blankstein R, Padera RF. Tumors metastatic to the heart. Circulation 2013;128:1790-4.

5. Bussani R, De-Giorgio F,Abbate A, et al. Cardiac metastases.J Clin Pathol 2007;60:27-34.

6. Butany J, Leong SW, Carmichael K, et al. A 30-year analysis of cardiac neoplasms at autopsy. Can J Cardiol 2005;21:675-80.

7. Kasama K, Ichikawa $Y$, Suwa $Y$, et al. Late cardiac metastasis from colorectal carcinoma 15 years after surgery. Asian Cardiovasc Thorac Ann 2016;24:66-8.

8. Laigle-Donadey F, Taillibert S, Mokhtari K, et al. Dural metastases. J Neurooncol 2005;75:57-61.

9. George KJ, Lau A, Ellis M, et al. Metastatic coagulopathic subdural hematoma: a dismal prognosis. Surg Neurol Int 2012;3:60.

10. Julson JR, Weiland T, Kemp WL. Acute subdural hemorrhage 
associated with both metastatic adenocarcinoma of the dura and minor head trauma: a case report and review of the literature. Acad Forensic Pathol 2018;8:769-76.

11. Fronczek J, Hollingbury F, Biggs M, et al. The role of histology in forensic autopsies: is histological examination always necessary to determine a cause of death? Forensic Sci Med Pathol 2014;10:39-43.

12. De-Giorgio F, Vetrugno G. Does the principle of cost/benefit analysis apply to forensic pathology? Am J Forensic Med Pathol 2010;31:300. 